\title{
Structure of the Canes Venatici I cloud of galaxies
}

\author{
Dmitry I. Makarov, Lidia N. Makarova and Roman I. Uklein \\ Special Astrophysical Observatory, Russian Academy of Sciences, Nizhnii Arkhyz, 369167 \\ Russia \\ email:dim@sao.ru,lidia@sao.ru,uklein@sao.ru
}

\begin{abstract}
We study the spatial distribution of the sparse cloud of galaxies in the Canes Venatici constellation. We determined distances of 30 galaxies using the tip of the red giant branch (TRGB) method. This homogeneous sample allows us to distinguish the zone of chaotic motions around the center of the system. A group of galaxies around M94 is characterized by the mass-luminosity ratio of $M / L_{B}=159(M / L)_{\odot}$. It is significantly higher than the typical ratio $M / L_{B} \sim 30(M / L) \odot$ for the nearby groups of galaxies. The CVn I cloud of galaxies contains 4-5 times less luminous matter compared with the well-known nearby groups, such as the Local Group, M 81 and Centaurus A. The central galaxy M 94 is at least 1 mag fainter than any other central galaxy of these groups. However, the concentration of galaxies in the Canes Venatici may have a comparable total mass.
\end{abstract}

Keywords. galaxies: distances and redshifts

Prominent fuzzy concentration of galaxies in the Canes Venatici constellation with lineof-sight velocity around $V_{\mathrm{LG}}=300 \mathrm{~km} \mathrm{~s}^{-1}$ forms so called the Canes Venatici I cloud (CVn I) of galaxies (see left panel of Fig. 1). This feature has been noted by Karachentsev (1966) and de Vaucouleurs (1975). This cloud clearly differs from the other nearby galaxy groups, such as the Local Group. CVn I shows absence of clearly prominent gravitational center and looks diffuse. This complex is mostly populated by dwarf galaxies of late morphological types.

We redefined the distances for 30 galaxies of CVnI using the deep images from the Hubble Space Telescope archive with the WFPC2 and ACS cameras. We carried out a high-precision stellar photometry of the resolved stars in these galaxies using the HSTphot (Dolphin 2000) and DOLPHOT (Dolphin 2002) software. Resulting color-magnitude diagrams allowed us to determine photometric distances by TRGB method using an advanced technique (Makarov et al. 2006) and modern calibrations (Rizzi et al. 2007). Comparison of our distance estimates with measurements by other authors (Karachentsev et al. 2002, Karachentsev et al. 2003, Karachentsev et al. 2006, Tully et al. 2006, Dalcanton et al. 2009) shows a very good agreement with a generally better accuracy.

M94 is a giant spiral galaxy located within the CVn I cloud and could be claimed as its gravitating center. The luminosity function of RGB stars in the M94 galaxy appears to be much more complex than that for the normal dwarf galaxies. It has a long, extended plateau near the cut-off. This is probably related with the complex history of star formation and metal enrichment in giant spiral M94. We estimated the distance modulus of $28.14 \pm 0.08 \mathrm{mag}$, which corresponds to the linear distance of $D=4.25 \pm 0.15 \mathrm{Mpc}$.

The Hubble diagram of nearby galaxies in the Canes Venatici is shown in right panel of Fig. 1. The new high precision of distance determinations allow us to identify an area of chaotic motions around the center of the system. The group of galaxies around M 94 is characterized by a median velocity of $287 \mathrm{~km} \mathrm{~s}^{-1}$, median distance of $4.28 \mathrm{Mpc}$, 

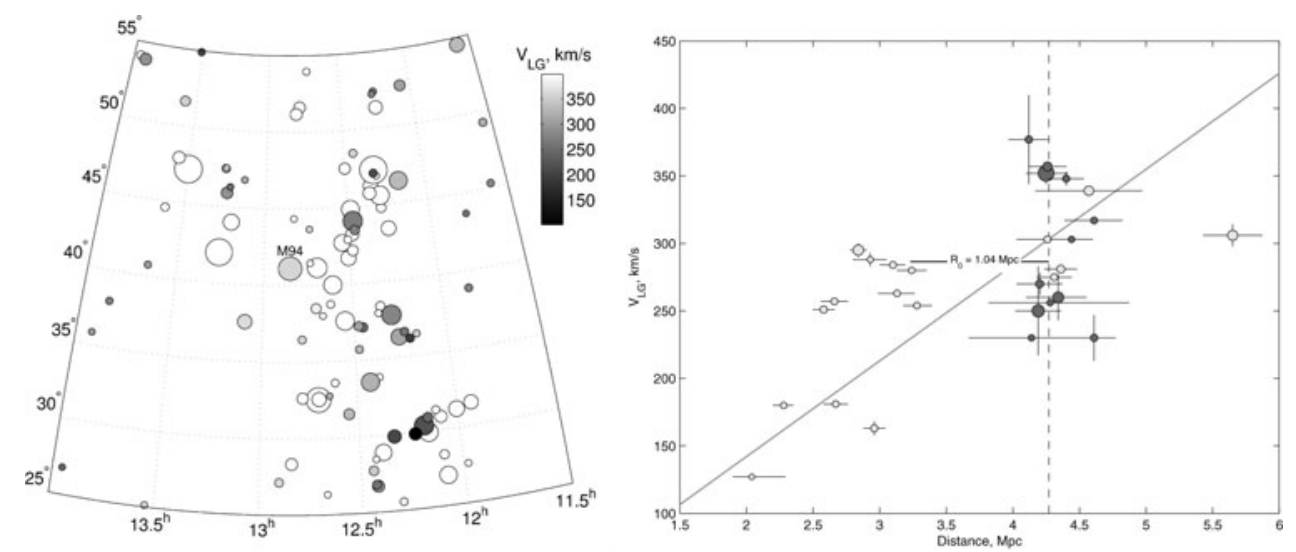

Figure 1. Left panel represents the distribution on the sky of galaxies in the Canes Venatici constellation. The distance-velocity diagram is shown on the right panel. The solid line corresponds to linear Hubble law with $H_{0}=71 \mathrm{~km} /(\mathrm{s} \times \mathrm{Mpc})$. The galaxies within $1 \mathrm{Mpc}$ from the center of the system are marked in dark gray.

and the total luminosity of $L_{B}=1.61 \times 10^{10} L_{\odot}$. The virial mass amounts to $M_{\text {vir }}=$ $1.93 \times 10^{12} M_{\odot}$, which corresponds to $M / L_{B}=120(M / L) \odot$. The projection estimation gives $M_{\text {proj }}=2.56 \times 10^{12} M_{\odot}$ and $M / L_{B}=159(M / L)_{\odot}$. If we assume that specific 'wave' on Hubble diagram in front of CVn I is result of gravitational attraction to the M 94 group of galaxies, we can estimate the radius of the zero velocity sphere of $1.04 \pm 0.15 \mathrm{Mpc}$. It corresponds to the mass $M_{\mathrm{R}_{0}}=2.38 \times 10^{12} M_{\odot}$.

Our mass-luminosity estimate, $M / L_{B}=120-160(M / L)_{\odot}$ for the CVn I cloud greatly exceeds the typical ratio $\sim 30$ for the nearby groups of galaxies, such as the Local Group $M / L_{B}=15-20(M / L)_{\odot}$ and M 81 group $M / L_{B}=19-32(M / L)_{\odot}$. Note that compared with the well-known nearby groups, such as the Local Group, M 81, and Centaurus A, the CVn I cloud contains 4-5 times less luminous matter, and M 94 is at least 1 mag fainter than any other central galaxy of these groups. However, the concentration of galaxies in the Canes Venatici may have a comparable total mass.

\section{Acknowledgements}

The authors thank prof. I. D. Karachentsev for constructive discussions. We are thankful for the support of the Russian Science Foundation grant 14-12-00965. We acknowledge the usage of the HyperLeda database (http://leda.univ-lyon1.fr).

\section{References}

J. J. Dalcanton, B. F. Williams, A. C. Seth, et al. 2009, ApJS, 183, 67

G. de Vaucouleurs, Nearby Groups of Galaxies (University of Chicago Press, Chicago, 1975), p. 557

A. E. Dolphin 2000, PASP, 112, 1383

A. E. Dolphin 2002, MNRAS, 332, 91

I. D. Karachentsev 1966, Astrophysics, 2, 39

I. D. Karachentsev, M. E. Sharina, D. I. Makarov, et al. 2002, A\&\&A, 389, 812

I. D. Karachentsev, M. E. Sharina, A. E. Dolphin, et al. 2003, A\& A, 398, 467

I. D. Karachentsev, A. Dolphin, R. B. Tully, et al. 2006, AJ, 131, 1361

D. Makarov, L. Makarova, L. Rizzi, et al. 2006, AJ, 132, 2729

L. Rizzi, R. B. Tully, D. Makarov, et al. 2007, ApJ, 661, 815

R. B. Tully, L. Rizzi, A. E. Dolphin, et al. 2006, AJ, 132, 729 